\title{
Benefit Design Innovations to Manage Specialty Pharmaceuticals
}

\author{
Debbie Stern, RPh
}

\begin{abstract}
BACKGROUND: Spending on specialty pharmaceuticals is rising faster than that for traditional drugs. In 2006, specialty drugs were the largest category driving drug costs and utilization trends. Even with effective management, expenditures on these agents are projected to increase exponentially in the coming years.
\end{abstract}

OBJECTIVE: To review benefit design strategies used by payers to control costs and manage utilization of specialty pharmaceuticals.

SUMMARY: The rapid growth in specialty pharmaceutical expenditures reflects the introduction of new agents, broader indications, and wider use in more common disease states. The true growth of the specialty pharmaceutical segment is obscured because many of these agents are reimbursed through the medical benefit, which often lacks the transparency necessary to accurately determine true cost and utilization trends. To date, efforts to control spending on biologics have been fragmented with most payers employing techniques for cost and utilization containment similar to those used for traditional pharmaceuticals. To ensure greater cost and utilization control, a benefit design that simultaneously provides optimal cost management, appropriate utilization, improved clinical management, enhanced clinical outcomes, and heightened patient safety should be established.

CONCLUSION: Current management techniques for specialty pharmaceuticals often represent a stop-gap approach for controlling rising drug costs. Creation of a specialty pharmacy benefit can optimize cost and utilization management.

J Manag Care Pharm. 2008;14(4)(suppl S):S12-S16

Copyright@ 2008, Academy of Managed Care Pharmacy. All rights reserved.

Author

DEBBIE STERN, RPh, is vice president, Rxperts, Inc., Irvine, California.

AUTHOR CORRESPONDENCE: Debbie Stern, RPh, Rxperts, Inc., 7700 Irvine Center Dr., Suite 800, Irvine, CA 92618. Tel.: 949.788.2909; Fax: 949.788.2979; E-mail: dstern@rxperts.net

\section{Introduction}

There are more than 250 specialty products currently on the market $^{1}$ and nearly 350 agents in late-stage trials. ${ }^{2}$ In 2006, Medco reported that spending on traditional pharmaceuticals increased by $2.8 \%$, whereas spending on specialty pharmaceuticals increased more than $16 \% .^{3}$ Similar trends were reported by Express Scripts, which noted that spending on specialty medications rose nearly $21 \%$ compared with only $6 \%$ for traditional drugs. ${ }^{4}$ Although eye opening, these reports only capture trends within the pharmacy, but not the medical benefit, where by some estimates, up to $60 \%$ of the drug spend is on specialty products. ${ }^{3}$ Consequently, trends published by even the largest pharmacy benefits managers (PBMs) only partially identify the true growth of spending on specialty products.

The growth in the specialty trend is dominated by higher costs for existing specialty agents, the introduction of new drugs to treat conditions where few treatment alternatives exist, new indications for existing products, and earlier and/or increased use of specialty products. Currently, the oncology therapeutic area-including oral and infused oncology drugsaccounts for more than half of the estimated $\$ 40$ billion spent each year on specialty products with spending on products to treat multiple sclerosis (MS), Hepatitis C, growth hormone deficiency, pulmonary arterial hypertension, asthma, and rheumatoid arthritis (RA) responsible for the balance. ${ }^{3}$ Several factors are expected to continue to put upward pressure on oral oncology specialty spending, including a rich pipeline containing more than 100 products (4 of which arrived in the market within the past year), a high degree of off-label use, and treatment regimens costing up to $\$ 10,000$ per month..$^{5}$ In addition, in many cases, cancers are often considered chronic conditions that can be medically managed over the long term. Thus, treatment cycles are longer as many of the newer more targeted agents are better tolerated and often used for prolonged maintenance treatment as monotherapy or in combination with specialty products.

\section{Traditional Pharmacy Benefits Management Techniques}

Predictably, the increased spending on specialty drugs has challenged payers to devise cost and utilization control strategies that simultaneously minimize costs and ensure appropriate utilization. Historically, payers have responded to the economic pressures of traditional drugs through the use of generic substitution, creation of formularies, maximization of manufacturer's rebates, therapeutic substitutions, quantity restrictions, and implementation of patient cost-sharing through the use of tiered copays and coinsurance. Similarly, utilization has been aggressively managed using prior authorization, step edits, and educating patients, providers, and purchasers.

With few exceptions, payers have attempted to adapt their existing drug benefit models rather than create entirely new 
benefits for specialty products. Currently, it appears that 2 features of the traditional benefit are at least partially effective when applied to specialty drugs: cost sharing and aggressive network management. However, other traditional control mechanisms are not quite applicable. For example, generic forms of specialty drugs are not available, and the number of "me-too" products is minimal, thus ruling out the economic benefits of robust market competition, such as cost savings from generic and therapeutic substitution, creation of formularies, and rebate maximization. As a result, payers are looking at new benefit strategies.

\section{Recommended Payer Specialty Benefit Strategies Cost Perspective}

Although specialty drug benefit plans vary from payer to payer, they all share the primary goals of optimizing costs, equalizing benefits, improving clinical management, and ensuring appropriate use. Outside of negotiating a favorable acquisition cost and rebate, perhaps the most important step toward optimizing costs is limiting the number of distribution channels by mandating the use of a specific specialty pharmacy or network of specialty pharmacies. Approximately $50 \%$ of plans have closed their pharmacy networks and mandated the use of a single specialty distribution channel for drugs covered under the pharmacy benefit, and even fewer plans limit the distribution channel for drugs covered under the medical benefit (Figure 1). ${ }^{6}$ The remaining plans allow access to both the specialty and retail pharmacy networks, as well as permit physicians to buy-and-bill. Because use of a preferred specialty pharmacy provider can result in significant cost savings, plans are often looking for ways to gradually increase control over the specialty network. Many plans identify the specialty categories that represent the greatest target for cost savings and mandates that all products in these categories be obtained through a preferred provider. Frequently, the first category subjected to a mandate is self-administered injectables. However, whether due to physician pushback, regulatory issues, lack of resources to implement and enforce a mandate, or a combination of factors, the prevalence of closed networks is significantly lower within the medical benefit and other settings where utilization is difficult to manage (e.g., Medicare and Preferred Provider Organizations). ${ }^{6}$

In addition to the mandated use of specialty pharmacy providers, other cost optimization strategies involve increased patient cost-sharing. Examples of cost-sharing strategies include establishing a flat copay for all drugs, implementing tiered copays for preferred and non-preferred agents, and requiring coinsurance, with or without a maximum dollar amount out-of-pocket (OOP). While patients tend to remain compliant to therapy despite paying higher overall OOP costs, further data are necessary to determine if these measures limit access to the appropriate specialty therapies. ${ }^{7}$
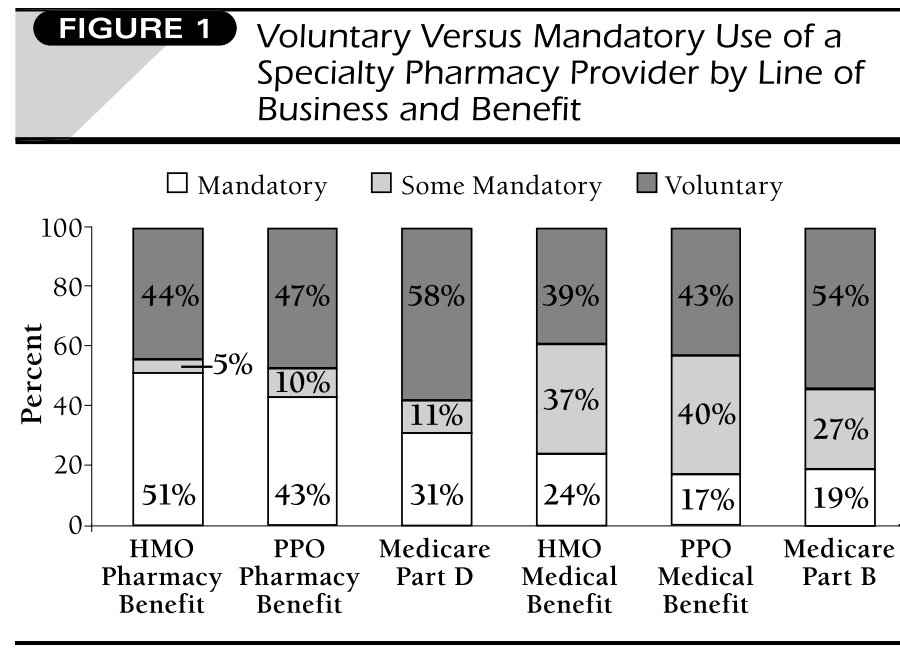

$\mathrm{HMO}=$ health maintenance organization; $\mathrm{PPO}=$ preferred provider organization .

\section{Benefit Perspective}

As mentioned, the design of the pharmacy benefit varies between plans, but the purpose of the benefit is consistent-it defines the terms and limits of the drug coverage provider by the third-party payer. More specifically, the drug benefit identifies the drugs and drug categories covered by the payer, the patient's share of the drug cost, the location where the drug can be prescribed, dispensed, and/or administered, and the quantity of the drug that will be covered. The benefit also spells out if the drug is subject to step edits, prior authorization, or other utilization controls.

Unlike traditional pharmaceuticals that are reimbursed under the pharmacy benefit, many office-administered specialty agents are covered as part of the medical budget. From a benefit design perspective, there appears to be a trend, albeit a slow one, to move all specialty drugs from the medical benefit into the pharmacy benefit. Generally, the applicable benefit is determined by the location in which the drug is administered. For example, infused medications (e.g., treatments for cancer and immune disorders) are more likely to be billed under the medical benefit, whereas self-administered therapies (e.g., treatments for MS and hepatitis C) are more commonly billed through the pharmacy benefit. However, because significant differences exist between the medical and pharmacy benefit design in terms of reimbursement rates, billing systems, patient cost-share, and clinical and utilization management techniques, integration of specialty agents into the pharmacy benefit raises complex cost, access, and administrative issues.

Differences between the design of the medical and pharmacy benefits may also lead to a misalignment of financial incentives among physicians, specialty pharmacy providers, and patients. Under the medical benefit, physicians can create a profitable revenue stream by controlling the selection of specialty drugs, 
specialty pharmacy providers can gain from the most profitable reimbursement schedules, and patients are attracted to the drug with the lowest copay. The inherent risk underlying all these conflicting incentives is that neither the health care providers nor patients are making sound clinical decisions unbiased by a financial motive. Thus, the onus is on the managed care industry to minimize the financial bias and implement a system that ensures the right drug is provided to the right patient at the right time.

From a payer's perspective, the ideal specialty benefit would be a single plan that encourages clinically sound and costeffective utilization. Although desirable, creating a unified specialty benefit would require a significant commitment to change on the part of multiple stakeholders within the organization (i.e., contracting, customer service, pharmacy department, medical management, legal, etc.) and would have implications across the entire enterprise. The reward for establishing a unified specialty benefit, however, could potentially be substantial. A single benefit would enhance data collection across the organization, allow the use of uniform coding (i.e., less worry about Healthcare Common Procedure Coding System [HCPCS] J-codes and National Drug Codes [NDC]), improve decision making around cost sharing, utilization and clinical management, improve contracting leverage, and allow for the implementation of well-established processes and controls.

Although attractive in many ways, arguments can also be made against the creation of a specialty benefit. There is the potential that a tightly controlled specialty benefit will negatively impact member and provider contracting as patients face increased OOP expenses with rising copays and cost sharing, while restrictions on buy-and-bill and mandates to use mandated specialty distribution methodologies, can alienate physicians. Additionally, employer groups that carve out pharmacy management to a PBM lack an internal pharmacy benefit in which to capture specialty prescriptions.

\section{Clinical and Utilization Management}

Patients using specialty drugs often require a significant amount of education on the disease itself, self-injection techniques, sideeffect management, and product handling and storage. Specialty pharmaceutical patients must also understand the importance of compliance with their prescribed treatment regimen. Many payers lack the capacity to provide the educational and clinical support programs necessary to maximize clinical outcomes and minimize the risk to patient safety, and often outsource these services to a specialty pharmacy provider.

\section{Developing Clinical Guidelines}

Payers are increasingly developing evidence-based clinical guidelines to ensure appropriate utilization, prevent waste and misuse of products (e.g., hemophilia, infertility, growth hormone), improve clinical outcomes of treatment, improve adherence to the prescribed regimen, and to ensure patient safety. Historically, health plans have developed clinical guidelines with little attention paid toward equalizing the multiple competing interests involved in selecting a proposed treatment strategy. To equilibrate this bias, a growing number of payers now mandate the involvement of members of the pharmacy and therapeutics or technology assessment committees in the guideline development process.

\section{Use of Prior Authorization}

In the absence of robust cost-effectiveness data on most specialty products, payers have implemented prior authorization (PA) requirements to ensure that physicians follow evidence-based practice guidelines and to ensure appropriate use of therapy. In addition to regulating utilization, a PA review every 6 months can be helpful to determine if a patient is experiencing an appropriate response to treatment based on achieving certain predetermined outcomes. PA also provides a system for tracking duration of therapy and for monitoring off-label or experimental usage. However, some patients fail to respond to therapy despite evidence-based treatment. In these cases, the challenge becomes identifying the appropriate next step. Should utilization of the drug continue to be authorized even if the predetermined outcomes are not achieved? Despite the lack of evidence of their overall efficacy, in many cases, plans ultimately pay for specialty agents because they represent the only therapeutic option for some diseases.

\section{Step Edits and Preferred Products}

Many payers are beginning to implement step edits to drive utilization to preferred drugs or to ensure the use of appropriate agents prior to initiating therapy with an injectable specialty product. According to a recent survey, $86 \%$ of plans designated 1 or more preferred specialty drugs, and $61 \%$ did so in 5 or more therapeutic categories. ${ }^{6}$ This trend, which is growing in popularity, is currently most common for therapeutics classes containing multiple agents, such as those used to treat MS, RA, hepatitis C, anemia, and growth hormone deficiency (Figure 2).

Because few specialty drugs are considered to be bio-equivalent despite belonging to the same therapeutic class, care needs to be exercised when selecting a preferred agent, as well as when determining access to alternative therapy. In several drug classes, the individual drugs within the drug class may have different indications. For example, a potential conflict can arise if infliximab has been selected as the preferred tumor necrosis factor (TNF) inhibitor, but a patient requires TNF inhibitor therapy for a condition not included in the infliximab label.

\section{Looking to the Future}

In the next 5 years, it is anticipated that specialty drugs will account for approximately $25 \%$ to $30 \%$ of total pharmacy costs. ${ }^{4}$ An analysis of future strategies for managing specialty pharmaceuticals indicates a trend toward requiring patients to pay more cost share via increasing the level of annual OOP maximums is very likely. ${ }^{8}$ Similarly, more payers will migrate specialty 


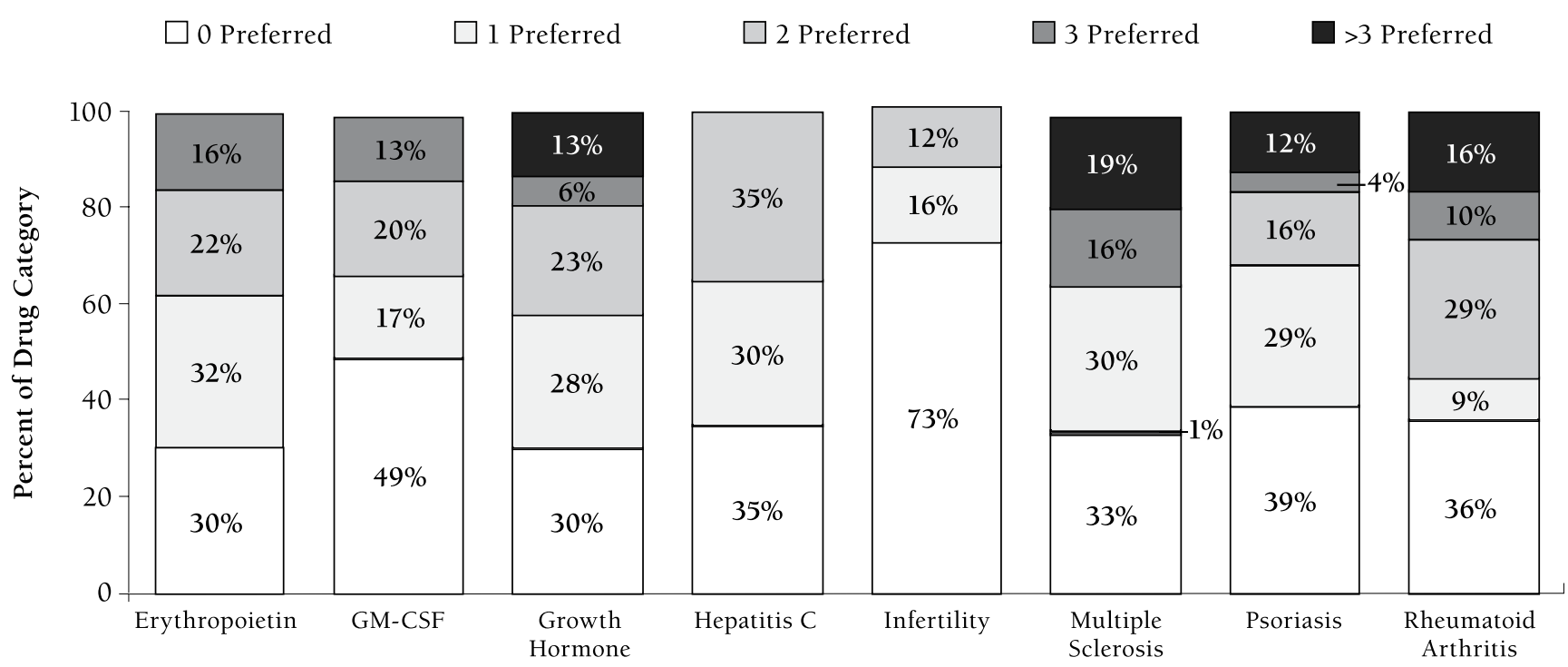

Therapeutic Category

pharmaceutical coverage to the pharmacy benefit or possibly create a separate benefit for specialty pharmacy.

\section{Specialty Vaccines}

Paralleling the growth of specialty pharmaceuticals is the emergence of several novel vaccines that can potentially be effective for the prevention of chronic disease. From a distribution standpoint, vaccines are typically office-administered agents covered under the medical benefit. However, due to their high cost and targeted therapeutic indications, they may also be considered specialty drugs. Payer strategies may drive the coverage of these agents to the pharmacy benefit, to maximize the cost and utilization management. Where these agents ultimately fall remains to be seen.

\section{Generic Biologics}

If available, generic biologics would introduce a degree of competition in the specialty market not currently present. However, the term "generic biologic" is actually somewhat misleading, as no 2 biologically engineered specialty products are truly equivalent. Terms such as 'biogeneric,' 'biosimilars,' and 'follow-on biologic' have been used in the United Sates and Europe, but currently there is no widely accepted definition of what actually constitutes a generic biologic. Introduction of generic biologics is also complicated by the current regulatory process, which lacks a pathway for generic biologic approval. In addition, the U.S. Food and Drug Administration (FDA) has no standard for proving generic versions of a specialty product are equivalent to the original branded agent. Congress will need to designate an approval pathway for these agents.

\section{Pharmacogenomics and Personalized Medicine}

There is growing interest in the use of genomics and proteinomics to guide diagnosis and treatment of disease. In fact, molecular diagnostics represents a $\$ 5$ billion market growing at an annual rate of $25 \%{ }^{9}$ Pharmacogenomic processes use molecular analysis to test for individual variations in genes, gene expression, proteins, and metabolites. The results of these tests can be used to guide treatment using drugs specifically designed to target the molecular mechanisms of the disease, but can also introduce several moral and ethical dilemmas for patients, employers, and health plans. Recently, a genetic test to evaluate a patient's suitability for warfarin treatment was introduced..$^{10}$ This next wave of the biotechnology revolution promises the ability to achieve optimum treatment outcomes or even alter an individual's predisposition for a disease, but not without a cost. To avoid being overwhelmed in the coming years, payers are encouraged to apply what they have learned regarding utilization management, benefit management, and cost containment of specialty agents and proactively apply it to pharmacogenomic products. 


\section{Dummary}

The combined impact of the exponential growth in the number of specialty pharmaceuticals, approval of new indications for existing products, earlier and/or increased use of specialty products, and the aging of the population guarantees that the costs associated with increased use of these agents will continue to rise in the years to come. Currently, many payers reimburse for these agents through both the medical and pharmacy benefit. However, to effectively manage cost and utilization, payers must implement a comprehensive specialty benefit design that guarantees that the right drugs are used by the right patients at the right time. Cost containment tactics inherent to a specialty benefit include assessing a uniform patient cost-share and mandated use of closed distribution networks by physicians. Utilization management tools include following evidence-based treatment guidelines and use of prior authorization to verify achievement of treatment endpoints. With the impending arrival of novel products, such as specialty vaccines, and the continued advances in the field of pharmacogenomics, it is imperative that payers begin to proactively plan their future specialty benefit designs.

\section{DISCLOSURES}

Debbie Stern, RPh, discloses that there was no relationship or financial interest related to the topic of this activity. Stern was responsible for the entire study concept and design of this article. She performed all the data collection, data interpretation, writing, and revision of this article.

\section{REFERENCES}

1. BIO (Biotechnology Industry Organization). Approved Biotechnology Drugs. Available at: www.bio.org/speeches/pubs/er/approveddrugs. Accessed July 10, 2007.

2. Butcher L. Newly final CMS policy may help plans when evidence for coverage is lacking. Biotechnol Healthcare. 2006;3:41-45

3. Medco Drug Trend Report 2007. Available at: www.drugtrend.com. Accessed October 12, 2007.

4. Express Scripts Drug Trend Report 2007. Available at: www.expressscripts.com/ourcompany/news/industryreports/drugtrendreport/2006/ dtrFinal.pdf. Accessed January 12, 2008.

5. PhRMA. Medicines in Development. Available at: www.phrma.org/ medicines_in_development/. Accessed March 27, 2008.

6. EMD Serono Injectables Digest 3rd Edition, 2007. Available at: www.emdserono.com. Accessed September 28, 2007.

7. Goldman DP, Joyce GF, Lawless G, Crown WH, Willey V. Benefit design and specialty drug use. Health Aff. (Millwood). 2006;25:1319-31.

8. Silverman E. Copayments: Too much, yet not enough? Biotechnol Healthcare. 2006;2:21-26.

9. Wyllie MG. Diagnostic potential unmasked. BJU Int. 2004;93:627-28.

10. U.S. Food and Drug Administration. FDA News. FDA Clears Genetic Lab Test for Warfarin Sensitivity. Available at: www.fda.gov/bbs/topics/ NEWS/2007/NEW01701.html. Accessed March 27, 2008. 